\title{
Giovanni Fabrizio Bignami
}

\author{
Giovanni Fabrizio Bignami, 'Nanni' to his friends, suddenly passed away on 25 May 2017. A pioneer in the \\ field of gamma-ray astrophysics, he identified Geminga: a mysterious, radio quiet, gamma-ray pulsar. \\ He was an undisputed leader, manager, maestro, colleague and friend.
}

T he international community lost a driving force for high-energy astronomy with the death of

Nanni Bignami. He spent his life fostering the realization of new, large-scale facilities to further our understanding of the Universe. His scientific interests spanned the full electromagnetic spectrum, from the radio wavelengths - with his recent coordination activity as chairman of the Square Kilometre Array international board - to X-rays and gamma-rays. He was the chair of the European Space Agency (ESA) committee that selected INTEGRAL, the largest gamma-ray observatory in space, and more recently was a charismatic member of the senior committee that selected Athena, the largest X-ray observatory ever conceived, to be launched by the ESA at the end of the next decade.

Nanni Bignami initiated his work in science by making use of data from the first sensitive gamma-ray satellites SAS-2 (NASA) and COS-B (ESA) - and playing a major role in the identification of Geminga - a new type of radio-quiet but gamma-ray-bright pulsar. His work on Geminga led to him becoming the first Italian to be awarded the Bruno Rossi Prize of the American Astronomical Society in 1993. He was renowned for his studies of gamma-ray pulsars, the gamma-ray source population and the $511 \mathrm{keV}$ annihilation emission from the Galactic Centre. In the past decade, his interest focused on groundbased facilities to detect very-high-energy gamma-rays and he worked internationally to promote the realization of the new Cherenkov Telescope Array (CTA). The Italian participation in this international program has now been approved by the Ministry of Education, Universities and Research, with Bologna selected as the headquarters for the CTA.

Nanni was also very active in planetary sciences: as president of the Italian Space Agency he sponsored participation in major ESA (ExoMars, BepiColombo, Mars Express) and NASA (Cassini, Dawn, Juno) missions. He greatly contributed to the success of several European and US astrophysical space missions: XMM-Newton (launched in 1999), INTEGRAL (2002), Swift (2004),

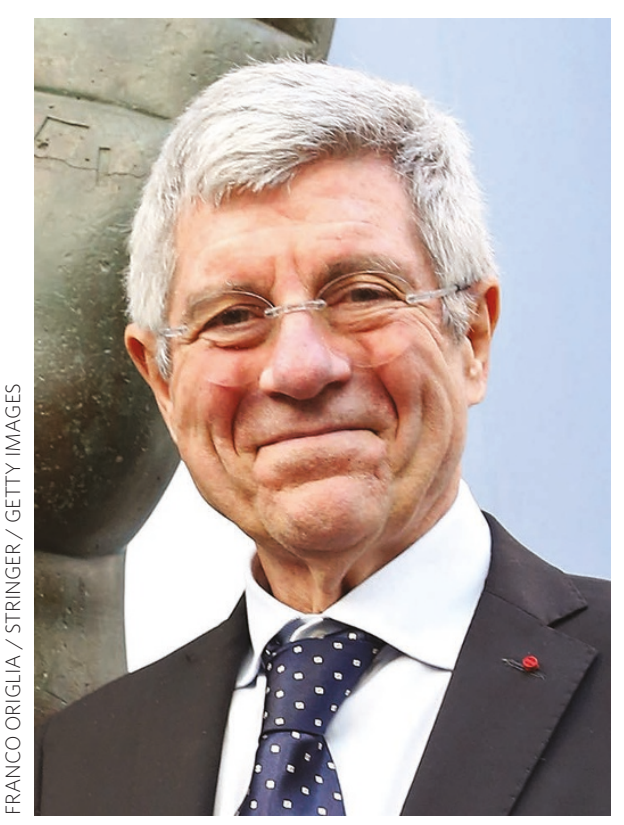

Giovanni Fabrizio Bignami (1944-2017)

the Italian small satellite AGILE (2007) and Fermi (2008), all of them still producing outstanding scientific results. He was always interested in space research, contributing to the first ESA satellite (COS-B). The culmination of his involvement in space science was achieved with his appointment as principal investigator (1988-1997) of the XMM-Newton X-ray observatory, a cornerstone mission of the ESA. Bignami led XMM-Newton from its inception, through development and launch. More than 18 years after its launch in 1999 , this observatory is one of the ESA's most scientifically productive endeavours. The day after his untimely passing, he should have been in a meeting in Madrid at the European Space Astronomy Centre to celebrate the 5,000 XMM-Newton refereed publications.

Nanni was born to lead: he started as a physics student at Milan University under the supervision of Giuseppe Occhialini, and spent time at Imperial College London. Just after receiving his diploma he was compulsorily enrolled in the Italian Army in the Alpine force, as an officer. He served as scientific director of the Italian
Space Agency (1997-2002), president of the ESA's Space Science Advisory Committee (2003-2006), coordinator of the Cosmic Vision 2015-2025 plan, director of the Centre d'Etude Spatiale des Rayonnements (2003-2006), president of the Italian Space Agency (2007-2008), president of COSPAR (Committee on Space Research) (2010-2014), president of the Italian National Institute for Astrophysics (2011-2015), and other international bodies. His leadership style was charismatic rather than authoritarian as he was constantly aware of the responsibilities that come with such positions. He was always open to discussion and willing to reconsider his position based on well-grounded views and suggestions, an important trait of a leader.

Nanni was appointed as professor in 1990 with the chair of Fisica Generale at Cassino University, in Italy, and since 2006, he was chair of Astronomia at the Istituto Universitario di Studi Superiori, in Pavia. In May 2000, he was appointed Officier de l'Ordre National du Mérite and in April 2006 as Officier de la Legion d'Honneur of the French Republic for the scientific merit of his work. Bignami was a member of the Italian Accademia Nazionale dei Lincei. In 2002, jointly with COSPAR, he was awarded the Harrie Massey Medal and Prize "for leadership in space science".

Among his many professional activities, as president of COSPAR he fostered the creation of Life Sciences in Space Research in 2013, with the first issue appearing in early 2014, now a well-regarded journal in its field. During his term as president, he reinforced the international leadership of COSPAR, started by his predecessor Roger Bonnet, by appointing a number of working groups that generated new ideas in different scientific fields, produced a number of roadmaps and ultimately had an important role in the inception of future space missions and observatories. Among them, the roadmaps Future of Space Astronomy (Adv. Space Res. 50, 1-55; 2012), Understanding Space Weather to Shield Society (Adv. Space Res. 55, 2745-2807; 2015), and the more recent Exoplanets (which is still in progress) stand out. He received several prestigious prizes: the 
von Karman Award of the International Academy of Astronautics in 2002, the Blaise Pascal Medal of the European Academy of Sciences in 2010 and the honour award as Officer of the Ordine al Merito of the Italian Republic in 2016. Recently, the asteroid 6852 Nannibignami was named after him.

He was a very successful science communicator, collaborating with the most important Italian newspapers such as Corriere della Sera, Il Sole 24 Ore, La Stampa, Il Messaggero and La Repubblica. He was an opinion maker, writing for magazines like
Le Scienze, WIRED, Vanity Fair, L'Espresso as well as the International Herald Tribune, Nature and Science. In parallel with his prolific scientific career, he published twelve books, some of which have been translated into seven different languages. He won several awards as a publicist and disseminator of scientific culture via television and other international media.

Nanni was a charming and theatrical speaker with deep classical knowledge, able to quote Latin better than any newly graduated student in classical languages.
Master of several languages, he was fluent in English, French, Italian and Russian. He was an undisputed international leader who was always thinking about scientific progress and the betterment of the scientific community. The international astronomical community has lost a leader.

\section{PIETRO UBERTINI}

Pietro Ubertini is at the Institute for Space Astrophysics and Planetology, Roma, Via Fosso del Cavaliere 100, 00133 Roma, Italy. e-mail:Pietro.ubertini@iaps.inaf.it 